\title{
Managing the Security-Energy Tradeoff in Distributed Cyber-physical Systems
}

\author{
Anh-Duy $\mathrm{Vu}$ \\ McMaster University, Canada \\ vud1@mcmaster.ca
}

\author{
Ramy Medhat* \\ University of Waterloo, Canada \\ rmedhat@uwaterloo.ca
}

\author{
Borzoo Bonakdarpour \\ Iowa State University, USA \\ borzoo@iastate.edu
}

\begin{abstract}
In this paper, we propose a technique that attempts to control energy consumption in distributed cyber-physical systems (CPS) in order to improve the level of security of the system. This is in contrast to most existing methods, where the system is set to use a certain level of authentication at design time, such as basic authentication, certificate-based authentication, or no authentication at all. To this end, we propose a notion of authenticatable task graph, which encodes standard task dependencies and allows for authentication tasks to be intermittently inserted into the computation task graph. The optimization objective here is to maximize the number of authentication tasks as well as peer-authentication, while remaining in the system energy bounds. We propose three offline optimization techniques and one online algorithm, where the system can dynamically manage the tradeoff between energy consumption and the level of security in the presence of uncertainties imposed by the physical environment. Our optimization algorithms are validated by a rich set of simulations as well as a real-world case study on a group of unmanned aerial vehicles (UAVs) that are assigned area search tasks and are required to perform peer-authentication within their battery limits.
\end{abstract}

\section{CCS CONCEPTS}

- Computer systems organization $\rightarrow$ Embedded and cyberphysical systems; • Security and privacy $\rightarrow$ Distributed systems security; Software and its engineering $\rightarrow$ Real-time schedulability.

\section{KEYWORDS}

Cyber-physical systems, security, energy consumption, optimization

\section{ACM Reference Format:}

Anh-Duy Vu, Ramy Medhat*, and Borzoo Bonakdarpour. 2019. Managing the Security-Energy Tradeoff in Distributed Cyber-physical Systems. In 10th ACM/IEEE International Conference on Cyber-Physical Systems (with CPS-IoT Week 2019) (ICCPS '19), April 16-18, 2019, Montreal, QC, Canada. ACM, New York, NY, USA, 10 pages. https://doi.org/10.1145/3302509.3311051

\section{INTRODUCTION}

Consider a fleet of autonomous unmanned aerial vehicles (UAVs) that carry out a joint mission, such as search and rescue. The fleet is

\footnotetext{
* Currently at Google-Waterloo, Canada.

Permission to make digital or hard copies of all or part of this work for personal or classroom use is granted without fee provided that copies are not made or distributed for profit or commercial advantage and that copies bear this notice and the full citation on the first page. Copyrights for components of this work owned by others than the author(s) must be honored. Abstracting with credit is permitted. To copy otherwise, or republish, to post on servers or to redistribute to lists, requires prior specific permission and/or a fee. Request permissions from permissions@acm.org.

ICCPS '19, April 16-18, 2019, Montreal, QC, Canada

(c) 2019 Copyright held by the owner/author(s). Publication rights licensed to ACM. ACM ISBN 978-1-4503-6285-6/19/04 ..\$15.00

https://doi.org/10.1145/3302509.3311051
}

obviously constrained by energy limits and a successful completion of the mission depends on rigorous path planning as well as accurate on-the-fly reporting of observations by the individual UAVs. Now, if one of the UAVs is compromised and makes false positive or negative reports, it may either cause premature completion of the mission or make it unnecessarily long, possibly beyond the current energy limits of the fleet. As another scenario, consider a wireless sensor network that is monitoring a large remote area to prevent massive wildfires. A sensor that detects smoke sends a message to the neighboring sensors and this message is propagated across the network to inform a base station for proper action. If one of these sensors is hacked, false positives can unnecessarily drain the network energy and false negatives can suppress an accurate reporting.

These examples clearly show that security of distributed cyberphysical systems (CPS) has to be an integral requirement in modern network-connected deployments, especially when they are safetyor mission-critical. Societies and public services are becoming vitally dependent on proper operation of CPS, especially with the rapid integration of the Internet of Things (IoT) with homes and public areas. Networks of mobile devices are also pervasive in many environments, where they are easily accessible and, therefore, exposed to greater threats. Moreover, battery-powered CPS usually impose constraints on energy consumption that could potentially affect the level of security maintained while executing functional tasks. Hence, distributed CPS should be designed to maximize the level of confidence in task results in the presence of cyberattacks, while reducing energy consumption to extend battery life.

Currently, most distributed CPS define a pre-specified level of security that the system should provide. The system is set to use a certain level of authentication at a predefined frequency. Such decisions do not account for different physical environments the system can be deployed into, different time-and-space-varying risks, and different threat/vulnerability-levels that call for different measures for security. Moreover, most current systems treat security as a non-functional property and a decorator for the main functional behavior of the system, and do not account for how security decisions could affect the control path that the distributed system takes. We are only aware of the work in $[7,12]$ that attempt to balance the system security vs. quality of control and service.

In this paper, we tackle this problem by introducing a notion of peer-authentication, where different components in a distributed CPS verify the output of each other to gain an acceptable global level of confidence. Obviously, providing more security (e.g., frequent authentication or key exchange) results in resource consumption (e.g., energy and communication bandwidth) at higher rates. Thus, one has to reach a balance between resource consumption and the level of security guarantees. We propose a general-purpose model that captures the interactions of the security vs. energy tradeoff in distributed CPS and allows system designers to parameterize the tradeoffs in their system. In particular, we consider a global task dependency graph partially executed by distributed nodes. Then, 


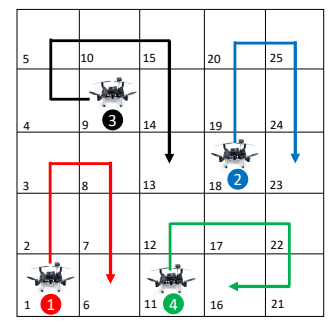

(a) No authentication.

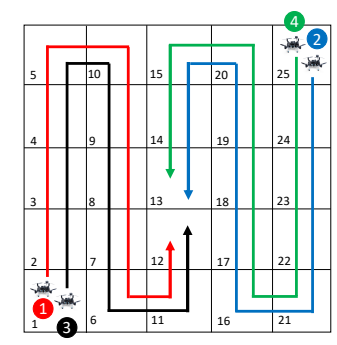

(b) Extreme authentication.

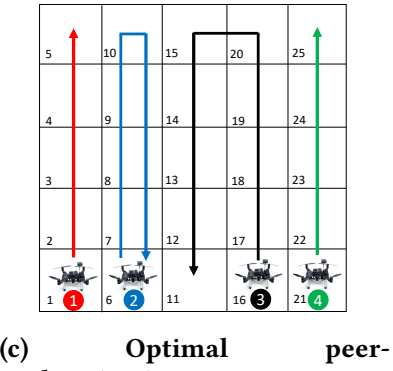

authentication.

Figure 1: Three peer-authentication scenarios in a multiUAV network.

we augment the task graph with authentication tasks. These tasks can be intermittently and arbitrarily inserted into the computation task graph. When execution units (e.g., CPU cores, sensors, UAVs, etc) execute more authentication tasks, a higher security confidence is gained and, in turn, more energy is consumed. Thus, our main objective is to determine when to schedule authentication and which nodes should be involved such that we reach a desired global termination state while operating under per-node energy constraints.

To better explain the idea of optimal peer-authentication, consider again our multi-UAV search and rescue application. Let us assume that searching each grid cell is a computation task and UAVs in neighboring cells can verify observations of their peers. Figure 1a shows a $5 \times 5$ grid area divided to four separate areas and each UAV operates in one of the independent areas with no peer-authentication. The total number of cells traveled by the four UAVs in this scenario is 25 . On the contrary, in Fig. 1b, extreme authentication is enforced by flying two UAVs over each grid cell at all times in two different altitudes. That is, one UAV always verifies the observations of the other one. The total number of cells traveled by the four UAVs in this scenario is 50 . In Fig. 1c, if we assume that all UAVs have equal energy and the global energy limit is sufficient to explore 30 cells, then the optimal peer-authentication strategy is that UAV 1 (red) and 2 (blue) cover cells $1-5$ and 6 10 , respectively, and can perform peer-authentication, as they fly concurrently in neighboring cells. Likewise, UAV 3 (black) and 4 (green) cover cells $11-20$ and $21-25$, respectively, and can perform peer-authentication. Then, UAV 2 returns to cell 6 , while UAV 3 starts exploring cells $11-15$ and can perform peer-authentication, as they fly in close proximity.

In order to find optimal peer-authentication strategies in our general-purpose model, we investigate four offline and online techniques:

- First, we propose an offline optimization method that produces a schedule of computation tasks intermittent with authentication tasks. This method is based on transforming our security vs. energy tradeoff optimization problem into mixed integer linear programming (MILP). This technique produces the optimal result, though is limited to small problem sizes.

- Next, we introduce two scalable offline algorithms that find sub-optimal solutions. The first algorithm is a non-trivial greedy technique, where execution units run and authenticate the tasks with the least energy requirements. To ensure that we complete all tasks in the graph, the algorithm checks to see if there is sufficient energy to execute all remaining computation tasks without authentication. At the cutoff point, the algorithm will abandon authentication and execute all remaining computation tasks only. We also propose a genetic algorithm that generates a random population (i.e., sequence of task executions). The population is then evolved by mating, mutating, selecting its individuals. Upon reaching the terminating condition, the best individual in the population is selected as the final solution to the problem.

- Since physical environments inherently bring uncertainties in a CPS, offline solutions are often not able to react properly to new energy and/or security conditions on the ground. In our example, weather conditions may change the rate of energy consumption, making the pre-planned flight and peer-authentication paths infeasible in reality. Thus, we propose an online algorithm that schedules tasks dynamically according to current energy limits of execution units by using backtracking to the last feasible step and resolving the optimization problem.

We have fully implemented our algorithms and report results of simulation as well as experiments on a real network of UAVs. Our simulations show that our genetic algorithm consistently outperforms the greedy algorithm in terms of executing more authentication tasks and better utilizing the remaining energy of execution units, while the MILP-based technique clearly finds the optimal solutions. We also develop a proof of concept by deploying our algorithms in a real network of UAVs that carry out a joint search mission. Our experiments show that in reality, UAVs consume $\% 12$ more energy on average than what flight paths obtained by an offline algorithm. This observation clearly motivates the idea of our online algorithm to operate in real CPS. We also present the results of experiments for our online algorithm in a scenario, where UAVs consume more energy than predicted, and how the algorithm successfully navigates them to complete their mission within their real limits, while maintaining the best level of security.

Organization. The rest of the paper is organized as follows. Preliminary concepts are presented in Section 2. In Section 3, we formally state our security vs. energy consumption tradeoff optimization problem. In Section 4, we introduce the transformation into MILP. Sections 5, 6, and 7 present our greedy, genetic, and online algorithms, respectively. Section 8 presents the simulation and experimental results. Related work is discussed in Section 9. Finally, we make concluding remarks and discuss future work in Section 10.

\section{PRELIMINARY CONCEPTS}

In this section, we present the preliminary concepts including our notion of authenticatable task graphs. 


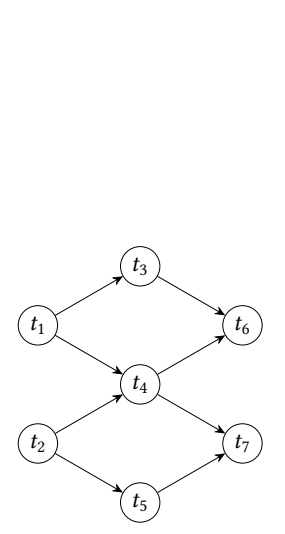

(a) Task graph.

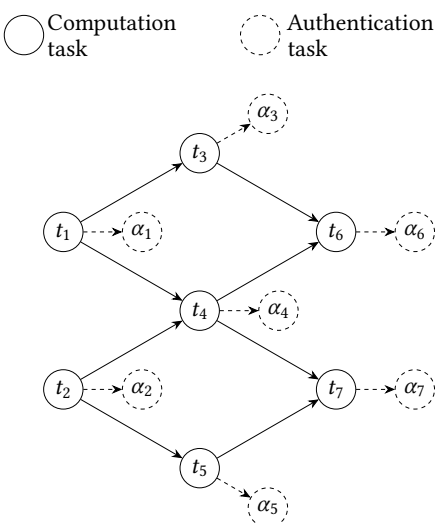

(b) An authenticatable task graph.
Figure 2: A task graph and its corresponding authenticatable task graph.

\subsection{Task Graph}

Definition 1. A task graph is a directed acyclic graph (DAG) $G=$ $(V, E)$, where each vertex $t \in V$ is a task, and each $\operatorname{arc}\left(t, t^{\prime}\right)$ in $E$ specifies an order, where task $t^{\prime}$ depends on $t$ (that is, task $t$ must be finished before task $t^{\prime}$ can be executed.)

In a task graph $G=(V, E)$, we denote the set of predecessors (respectively, successors) of a task $t$ by $P_{t}$ (respectively, $S_{t}$.) Formally,

$$
P_{t}=\left\{t^{\prime} \mid\left(t^{\prime}, t\right) \in E\right\} \quad S_{t}=\left\{t^{\prime} \mid\left(t, t^{\prime}\right) \in E\right\}
$$

An entry point is a task with no incoming edges and a termination point is a task with no outgoing edges. For example, Fig. 2a demonstrates a task graph of seven tasks, where $t_{1}$ and $t_{2}$ are entry points and $t_{6}$ and $t_{7}$ are termination points.

\subsection{Authenticatable Task Graph}

We now expand on Definition 1 by augmenting task graphs with means for authentication. We define two equal-size sets of correlated tasks:

- Computation tasks (denoted $\left.T=\left\{t_{1}, \ldots, t_{k}\right\}\right)$ that represent the actual work to be done. These are the tasks in Definition 1 (i.e., $T=V$ ).

- Authentication tasks (denoted $A=\left\{\alpha_{1}, \ldots, \alpha_{k}\right\}$ ) associated with computational tasks. An authentication task optionally follows the actual work and authenticates the output of a computational task.

Definition 2. Let $G=(V, E)$ be a task graph as defined in Definition 1 . The corresponding authenticatable task graph is a DAG $\mathcal{G}=(\mathcal{V}, \mathcal{E})$, where

$$
\mathcal{V}=T \cup A
$$

is the set of all tasks. The directed edges in the graph are as follows:

- For each edge $\left(v_{i}, v_{j}\right) \in E$, we include an edge $\left(t_{i}, t_{j}\right)$ in $\mathcal{E}$ to retain the dependency.

- There is an edge from a computation task to its corresponding authentication task. That is,

$$
\forall v_{i} \in V:\left(t_{i}, \alpha_{i}\right) \in \mathcal{E}
$$

Figure $2 \mathrm{~b}$ illustrates an authenticatable version of the task graph in Figure 2a. As can be seen in the figure, for every task $t$, there is a corresponding authentication task $\alpha$. The dashed arrows represent the edges from computation to their corresponding authentication tasks.

\subsection{Execution unit}

Intuitively, an execution unit is an entity that is able to execute tasks and is constrained by its energy capacity. For example, sensors or unmanned aerial vehicles (UAVs) are able to perform certain tasks within their energy limits and they consume some minimal energy when they are idle.

Definition 3. An execution unit $u=\left\langle\mathcal{A}_{u}, \mathcal{B}_{u}, \mathcal{I}_{u}\right\rangle$ is a tuple of three elements:

- The affinity set $\mathcal{A}_{u}$ is a function that maps $u$ to a set of tasks that $u$ is allowed to execute;

- Energy bound $\mathcal{B}_{u}$ is a non-negative real number indicating the upper bound on the energy of $u$;

- Idle energy consumption rate $\mathcal{I}_{u}(t)$ is a non-negative real number indicating the amount of energy consumed every second when the execution unit is idle, i.e. not executing any task.

We denote the set of all execution units by $\mathbb{U}$. An execution unit consumes time and energy when executing a task from the authenticatable task graph $\mathcal{G}$. There is also cost associated with transitioning from one task to another. For instance, for a CPU execution unit, there could be a sequence of tasks that cause cache misses if executed in a specific order, and that results in more time and energy consumed by the CPU. In the case of UAVs, there may be geographical separation between tasks and so the order of tasks determines the flight distance and consequently the required amount of time and energy to execute the task.

\subsection{Cost Functions}

To determine the costs of an execution unit $u \in \mathbb{U}$ executing tasks in an authenticatable task graph $\mathcal{G}$, we define the following cost functions:

- Execution cost $\xi: \mathbb{U} \times \mathcal{V} \rightarrow \mathbb{R}_{\geq 0}^{2}$ maps an execution unit $u$ and a (computation or authentication) task $v$ to a pair of real numbers $(\tau, \varepsilon)$, where $\tau$ and $\varepsilon$ are the amount of time and energy consumed by the unit executing $v$.

- Transition cost $\mathcal{T}: \mathbb{U} \times \mathcal{V}^{2} \rightarrow \mathbb{R}_{\geq 0}^{2}$ is a function that maps an execution unit $u$ and a pair of tasks $v, v^{\prime}$ to a pair of real numbers $(\tau, \varepsilon)$, where $\tau$ and $\varepsilon$ are the amount of time and energy consumed by the unit transitioning from $v$ to $v^{\prime}$.

\subsection{Schedule}

A schedule $\mathcal{S}$ is a function that determines which execution units run a task and when they start executing it. It is defined as follows:

$$
\mathcal{S}: \mathcal{V} \rightarrow 2^{\mathbb{U}} \times \mathbb{R}_{\geq 0}
$$

Thus, $\mathcal{S}$ maps a task in the set of vertices $\mathcal{V}$ in authenticatable graph $\mathcal{G}$ to a set of execution units $\mathbb{U}$ and a start time $s \in \mathbb{R}_{\geq 0}$.

Note that the schedule allows assigning a task to multiple execution units and only one start time. The purpose of this is to support authentication tasks. An authentication task is performed by two or more units concurrently. This allows us to model communication as part of the peer-authentication process such as key exchange. That is, peer-authentication should take place at the same time among 
peers. It also allows us to model corroboration of a task output such as partial replication of the computation or verification of digests. A computation task on the other hand can only be executed by one execution unit. We enforce these constraints in the next subsection.

Finally, an assignment $\lambda_{u}^{\mathcal{S}}$ is the ordered sequence of tasks executed by $u$ as determined in schedule $\mathcal{S}$. It is defined as $\lambda_{u}^{\mathcal{S}}=$ $\left(v_{0}, v_{1}, \cdots\right)$ such that

$$
\begin{gathered}
\forall v \in \lambda_{u}^{\mathcal{S}}: u \in \mathcal{S}\left(v_{i}\right) . U \\
\forall v_{i}, v_{i+1} \in \lambda_{u}^{\mathcal{S}}: \mathcal{S}\left(v_{i}\right) . s<\mathcal{S}\left(v_{i+1}\right) . s
\end{gathered}
$$

where $v_{i} \in \mathcal{V}$, and $\mathcal{S}(v) . U$ and $\mathcal{S}(v)$.s denote the set of units and start time mapped to $v$.

\section{PROBLEM STATEMENT}

We now introduce the formal statement of the problem.

\subsection{Problem Constraints}

We now detail the constraints of our proposed problem. Our goal is to design a schedule $\mathcal{S}$ subject to the following constraints.

Dependency. A schedule obeys the dependency encoded in $\mathcal{G}$, such that the following holds:

$$
\forall\left(v, v^{\prime}\right) \in \mathcal{E}: \mathcal{S}\left(v^{\prime}\right) . s \geq \mathcal{S}(v) . s+\max \{\xi(u, v) . \tau \mid u \in \mathcal{S}(v) . U\}
$$

That is, for every pair of tasks $v$ and $v^{\prime}$, where $v^{\prime}$ depends on $v$, the start time of $v^{\prime}$ is greater or equal to the termination time of $v$. The termination time of $v$ is calculated as the start time of $v$ plus the execution time of $v$ spent by its assigned execution unit as determined by the schedule.

Transition time. A schedule assigning a sequence of tasks to an execution unit must account for the transition time:

$$
\begin{aligned}
\forall u \in \mathbb{U}: & \forall\left(v_{i}, v_{i+1}\right) \in \lambda_{u}^{\mathcal{S}}: \\
& \mathcal{S}\left(v_{i+1}\right) . s \geq \mathcal{S}\left(v_{i}\right) . s+\xi\left(u, v_{i}\right) \cdot \tau+\mathcal{T}\left(u, v_{i}, v_{i+1}\right) . \tau
\end{aligned}
$$

Affinity. Every task is run by an execution unit allowed to run the task as determined by its affinity set $\mathcal{A}_{u}$ :

$$
\forall v \in \mathcal{V}: v \in \mathcal{A}_{\mathcal{S}(v) . U}
$$

where $\mathcal{A}_{\mathcal{S}(v) . u}$ is the affinity set of the execution unit assigned to run $v$ as determined by the schedule $\mathcal{S}$.

Completion. All computation tasks must be executed exactly once.

$$
\forall t \in T:|\mathcal{S}(t) \cdot U|=1
$$

Energy bound. Each execution unit should obey its energy bound. That is, for every execution unit, the total amount of energy consumed while executing an assignment is less than or equal to its bound. First, note that an execution unit can also be idle between tasks. For an assignment $\lambda_{u}^{\mathcal{S}}$, let $\iota\left(\lambda_{u}^{\mathcal{S}}, i\right)$ denote the idle time spent before executing the $i$ th task in the assignment:

$$
\iota\left(\lambda_{u}^{\mathcal{S}}, i\right)=\mathcal{S}\left(v_{i}\right) . s-\mathcal{S}\left(v_{i-1}\right) . s-\xi\left(u, v_{i-1}\right) \cdot \tau-\mathcal{T}\left(u, v_{i-1}, v_{i}\right) \cdot \tau
$$

Thus, the following constraint enforces that an execution unit obeys its energy bound:

$$
\forall u: \sum_{i=0}^{\left|\lambda_{u}^{\mathcal{S}}\right|-1} \xi\left(u, v_{i}\right) \cdot \varepsilon+\mathcal{T}\left(u, v_{i}, v_{i+1}\right) \cdot \varepsilon+\mathcal{I}_{u}\left(\iota\left(\lambda_{u}^{\mathcal{S}}, i+1\right)\right) \leq \mathcal{B}_{u}
$$

Peer authentication. As mentioned earlier, an authentication task is performed by two or more units concurrently. First, we enforce that two or more units participate in every authentication task as follows:

$$
\forall \alpha \in A:|\mathcal{S}(\alpha) \cdot U|=0 \vee|\mathcal{S}(\alpha) \cdot U| \geq 2
$$

That is, if an authentication task is ever executed, it must be executed by at least two peers. Second, we enforce that one of the execution units participating in authentication is the unit that performed the respective computation task. This allows us to model authentication that depends on the task's output, which is expected when corroborating the output or reaching consensus:

$$
\forall \alpha_{i} \in\{\alpha|| \mathcal{S}(\alpha) \cdot U \mid \neq 0\}: \mathcal{S}\left(t_{i}\right) . U \subset \mathcal{S}\left(\alpha_{i}\right) . U
$$

where $t_{i}$ is the respective computation task of $\alpha_{i}$. That is, for all authentication tasks that were executed per the schedule, the set of execution units that executed their respective computation tasks (which is only one unit as per Constraint (7) is a subset of the authentication peers.

\subsection{Optimization Objectives}

More authentication tasks implies higher confidence in task results. Our optimization objective is two fold:

- Maximize authentications. Our first objective is to maximize the number of authentication tasks executed by any execution unit. That is:

$$
\max |\{\alpha|| \mathcal{S}(\alpha) \cdot U \mid \neq 0\}|
$$

In this formula, every authentication task is counted once regardless of how many peers participated in authentication.

- Maximize peers. A higher number of peers participating in authentication also increases confidence and resilience to attacks. Thus, our second objective is to maximize the average number of peers per authentication task:

$$
\max \frac{\sum_{\alpha \in A}|\mathcal{S}(\alpha) \cdot U|}{|\{\alpha|| \mathcal{S}(\alpha) \cdot U \mid \neq 0\}|}
$$

\section{TRANSFORMATION TO MIXED INTEGER PROGRAMMING (MILP)}

As the first step to solve the optimization problem presented in Section 3, we transform it into a mixed integer linear program (MILP).

\subsection{Decision Variables}

Let $\mathcal{G}=(\mathcal{V}, \mathcal{E})$ be an authenticatable task graph, $\mathcal{T}$ be the cost function, and $\mathbb{U}$ be the set of all execution units. The following are decision variables in our MILP instance:

- $x_{i}^{u} \in\{0,1\}$ indicates whether an execution unit $u \in \mathbb{U}$ executes task $i$, where $i \in \mathcal{V}$.

- $y_{i} \in\{0,1\}$ indicates whether multiple execution units execute authentication task $i$, where $i \in A$.

- $x_{i j}^{u} \in\{0,1\}$ indicates whether an execution unit $u \in \mathbb{U}$ transitions from task $i$ to $j$, where $i, j \in \mathcal{V}$.

- $s_{i} \in \mathbb{R}_{\geq 0}$ is the start time of task $i$, for $i \in \mathcal{V}$. Note that since there is only one start time for each task, if an authentication task is executed by multiple units, it will be executed concurrently.

- $f^{u} \in \mathbb{R}_{\geq 0}$ indicates the time when execution unit $u \in \mathbb{U}$ finishes the last task in its assignment, for $i \in \mathcal{V}$.

- $l^{u} \in \mathbb{R}_{\geq 0}$ indicates the total amount of idle time spent by execution unit $u \in \mathbb{U}$ when processing its assignment. 


\subsection{Constraints}

This subsection details the constraints of the MILP instance based on the ones identified in Section 3.1.

Affinity. An execution unit can only execute tasks in its affinity set:

$$
\sum_{i \notin \mathcal{A}_{u}} x_{i}^{u}=0 \quad \text { for all } u \in \mathbb{U}
$$

Completion. Every computation task in the graph must be executed once:

$$
\sum_{u \in \mathbb{U}} x_{i}^{u}=1 \quad \text { for all } i \in T
$$

Dependency. First, we assume a dummy task $d_{s}$ which is utilized to connect all entry points in $\mathcal{G}$. Formally,

$$
\forall v \in\left\{v|| P_{v} \mid=0\right\}:\left(d_{s}, v\right) \in \mathcal{E}^{\prime} \wedge \sum_{u \in \mathbb{U}} \mathcal{T}\left(u, d_{s}, v\right)=(0,0)
$$

where $\mathcal{E}^{\prime} \supset \mathcal{E}$. Moreover, every execution unit has to initially execute $d_{s}$ with no time or energy cost. That is,

$$
\begin{array}{ll}
s_{d_{s}}=0 & \text { for all } u \in \mathbb{U} \\
x_{d_{s}}^{u}=1 & \text { for all } u \in \mathbb{U} \\
\xi\left(u, d_{s}\right) \cdot \tau=\xi\left(u, d_{s}\right) \cdot \epsilon=0 &
\end{array}
$$

Now we constrain a task's start time to enforce dependency:

$$
s_{j} \geq s_{i}+\sum_{u} x_{i}^{u} \times \xi(u, i) \cdot \tau \quad \text { for all }(i, j) \in \mathcal{E}^{\prime}
$$

Consecutiveness. We constrain that an assignment is a consecutive sequence which starts from $d_{s}$, goes through adjacent tasks and does not return to $d_{s}$. That is,

$$
\begin{array}{ll}
\sum_{j \in \mathcal{V}} x_{d_{s} j}^{u}=1 & \text { for all } u \in \mathbb{U} \\
0 \leq \sum_{i \in \mathcal{V} \cup\left\{d_{s}\right\}} x_{i j}^{u}-\sum_{k \in \mathcal{V}} x_{j k}^{u} \leq 1 & \text { for all } u \in \mathbb{U}, j \in \mathcal{V} \\
\sum_{i \in \mathcal{V}} x_{i d_{s}}^{u}=0 & \text { for all } u \in \mathbb{U}
\end{array}
$$

Transition time. Next, we ensure that the start time of tasks accounts for the transition time from the previous task:

$$
\begin{array}{r}
s_{j} \geq M\left(x_{i j}^{u}-1\right)+s_{i}+x_{i j}^{u} \times((\xi(u, i) \cdot \tau+\mathcal{T}(u, i, j) \cdot \tau) \\
\text { for all } i, j \in \mathcal{V}, u \in \mathbb{U}
\end{array}
$$

where $M$ is a large constant (larger than maximum possible start time) to cancel the constraint if unit $u$ does not execute $i$ then $j$.

Energy bound. Every execution unit honors its energy bound. To constrain energy consumption, we first add constraints to bound the finish time of execution units:

$$
f^{u} \geq M\left(x_{i}^{u}-1\right)+s_{i}+\xi(u, i) \cdot \tau \quad \text { for all } u \in \mathbb{U}, i \in \mathcal{V}
$$

Thus, $f^{u}$ is greater than or equal to the finish time of the last task that execution unit $u$ runs. Next, $l^{u}$ is the idle time spent by unit $u$ :

$$
l^{u}=f^{u}-\sum_{i \in \mathcal{V}} x_{i}^{u} \times \xi(u, i) \cdot \tau-\sum_{(i, j) \in \mathcal{V}^{2}} x_{i j}^{u} \times \mathcal{T}(u, i, j) \cdot \tau
$$

Finally, we constrain the energy consumption of execution unit $u$ as follows:

$$
\sum_{i \in \mathcal{V}} x_{i}^{u} \times \xi(u, i) . \epsilon+\sum_{(i, j) \in \mathcal{V}^{2}} x_{i j}^{u} \times \mathcal{T}(u, i, j) . \epsilon+l_{u} \times \mathcal{I}_{u} \leq \mathcal{B}_{u}
$$$$
\text { for all } u \in \mathbb{U}
$$

Peer Authentication. An authentication task must be executed by two or more execution units.

$$
\begin{aligned}
& \sum_{u \in \mathbb{U}} x_{i}^{u} \leq 0+y_{i} M \\
& \sum_{u \in \mathbb{U}} x_{i}^{u} \geq 2+\left(y_{i}-1\right) M \quad \text { for all } i \in A
\end{aligned}
$$

where $M>|\mathbb{U}|$.

Authentication after computation. An authentication task must follow its respective computation task in order to verify the output of the computation. Essentially, we want to ensure that the arc from a computation task to its respective authentication task in the authenticatable graph is visited by a unit participating in the authentication.

$$
\sum_{u \in \mathbb{U}} x_{i j}^{u} \geq y_{j} \quad \text { for all }(i, j) \in \mathcal{E} \wedge j \in A
$$

\subsection{Objective Functions}

As mentioned in Subsection 3.2, our problem requires optimizing multiple objectives. We use a hierarchical approach to model multiple objective functions. First of all, our main objective is to maximize the number of executed authentication tasks, regardless of the number of units participating in authentication. Hence, our first objective is as follows:

$$
\max \sum_{i \in A} y_{i}
$$

Upon solving the MILP given Objective (20), we add a constraint as follows:

$$
\sum_{i \in A} y_{i}=\mathbb{M}
$$

where $\mathbb{M}$ is the solution of the MILP with Objective (20). Based on the new constraint, we set the objective of the new MILP to the following:

$$
\max \sum_{u \in \mathbb{U}} \sum_{i \in A} x_{i}^{u}
$$

Thus, the new MILP maximizes the number of participants in authentication tasks given the number of executed authentication tasks is $\mathbb{M}$.

\section{GREEDY ALGORITHM}

In this section, we introduce our greedy algorithm (Algorithm 1). The greedy choice in the algorithm is running and authenticating the tasks with the least energy requirements. To ensure that we complete all tasks in the graph, the algorithm checks to see if there is available energy to execute all remaining computation tasks without authentication. At the cutoff point, the greedy algorithm will abandon authentication and execute all remaining computation tasks only.

Our algorithm implements the Nearest Neighbour Heuristic (NNH) (i.e., computation tasks are sequentially chosen according to their energy requirements) to check whether there are feasible 


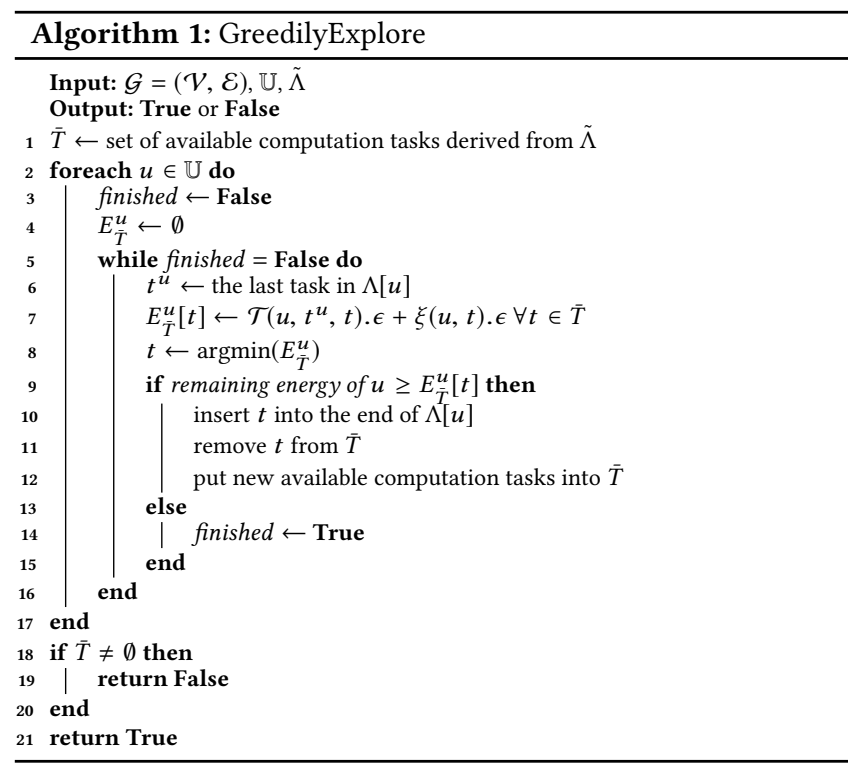

assignments to execution units that result in the completion of all remaining tasks. It takes as input an authenticatable task graph $\mathcal{G}$, a set of execution units $\mathbb{U}$, and the partially built assignments to the units $\tilde{\Lambda}$. The algorithm works as follows:

(1) Determine the set of available computation tasks $\bar{T}$ (i.e., the set of computation tasks that have not been executed, and whose all predecessors have been completed) (Line 1).

(2) Utilize a loop to sequentially extend the assignment of each unit (Lines 2 -17). At each iteration, (i) choose the cheapest task to execute (Line 8), (ii) if the unit has enough energy to do the task, append the task to the end of the unit's assignment (Line 10) which potentially unlock other tasks that were waiting for their precedents to complete. These tasks can now be added to $\bar{T}$ (Line12), and (iii) otherwise, switch to the next execution unit (Line 14).

(3) If at the end of the loop there are still unexecuted tasks, this indicates a failure to explore the graph with the available energy. Otherwise, succeed.

Next, we introduce Algorithm 2, which is the main algorithm that greedily maximizes the number of $M$-peered authentication tasks, where $M \geq 2$ is an input. The algorithm works as follows:

(1) Initialize the assignments of execution units with $d_{s}$ (Line 1). Recall that $d_{s}$ is the dummy start task introduced in Section 4.

(2) Initialize the set of available tasks, $\bar{T}$, with the set of entry points in the given $\mathcal{G}$ (Line 2.)

(3) Utilize NNH to pick the cheapest computation task $t$ for an execution unit (Line 9), which is similar to what is done in Algorithm 1.

(4) Gradually pick the cheapest peer to participate in the authentication of $t$ (Line 21). Check whether there are feasible assignments to the remaining tasks using Algorithm 1 (Line 23): If there is enough energy, we commit the peer (Line 24), update the assignments (Line 25), and try to add another peer. If there are no more peers to add or we have reached the maximum number of peers, go back to step 3 .

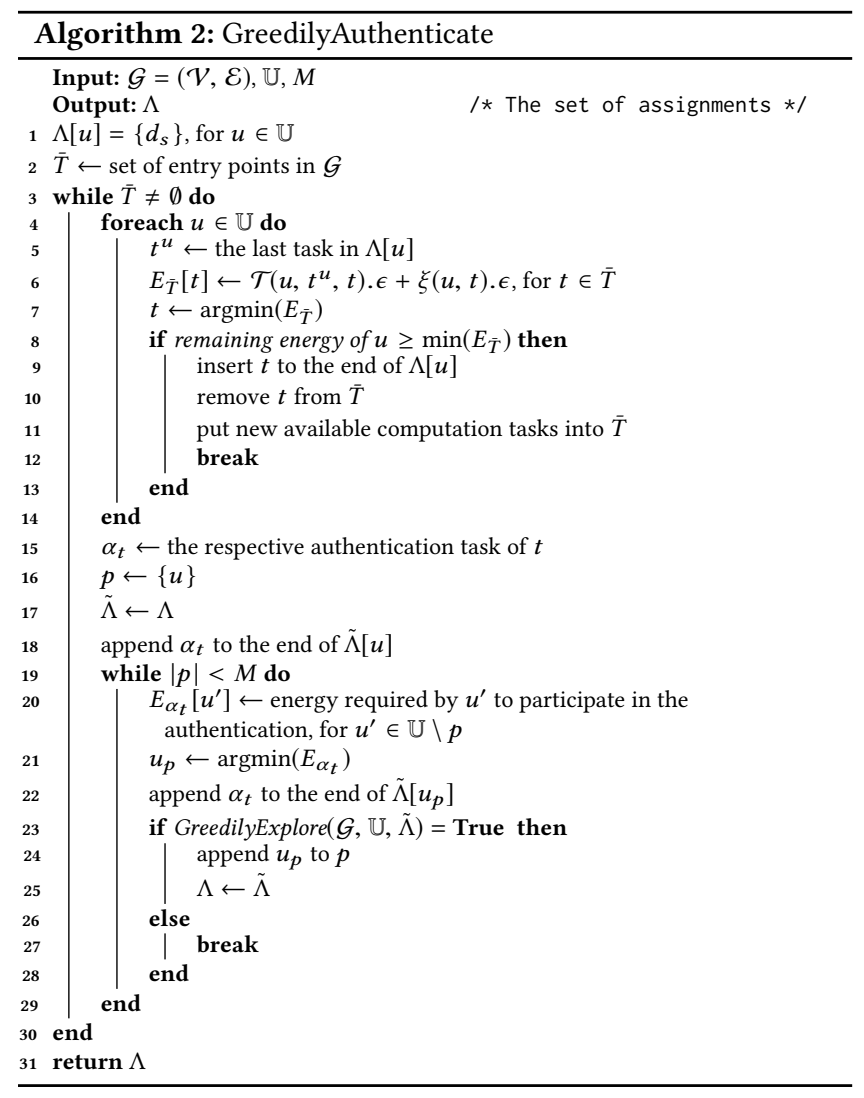

\section{GENETIC ALGORITHM}

In this section, we present our proposed genetic algorithm (GA). The challenge in designing a GA to solve our problem is twofold: (1) authentication tasks not only are optional but also require two or more execution units to start executing concurrently, and (2) the problem has two competing objectives which significantly reduce the convergence rate. To address these challenges, we first utilize a 3-part-string chromosome to encode a solution and propose the corresponding mating, mutating and selecting methods.

\subsection{Representation of Solution}

Each chromosome consists of:

- The Selected Authentication Tasks (SAT) string contains $|\mathbb{U}|$ substrings. Each denoted by $S A T_{u}$, for each $u \in \mathbb{U}$, is a binary vector of length $|A|$, such that $S A T_{u}(i)=1$ indicates that execution unit $u$ participates in authentication task $\alpha_{i}$.

- The Computation Task Assignment (CTA) string is a vector of length $|T|$, such that $C T A(i)=j$, where $0 \leq i \leq|T|$, and $0 \leq j \leq|U|$; i.e., computation task $t_{i}$ is assigned to unit $u_{j}$.

- The Schedule String (SS) is a vector of length $|\mathcal{V}|$, such that $S S(i)=\left(t, s_{t}\right)$, where $0 \leq i \leq|\mathcal{V}|, t \in \mathcal{V}$, and $s_{t}$ is the start time of task $t$. Moreover, the vector is also a topological sort [2] of the authenticatable graph (i.e., the total ordering of tasks in the vector satisfies the precedence constraints.)

A chromosome is represented by a tuple $\langle S A T, C T A, S S\rangle$. Figure 3 illustrates a chromosome for the authenticatable task graph in Figure $2 \mathrm{~b}$ and 2 execution units. For simplicity, we only show tasks in 


\begin{tabular}{|c|c|c|c|c|c|c|c|c|c|c|c|c|c|c|}
\hline & \multicolumn{7}{|c|}{$S A T_{1}$} & \multicolumn{7}{|c|}{$S A T_{2}$} \\
\hline$S A T$ & 1 & 0 & 0 & 1 & 0 & 1 & 0 & 1 & 0 & 0 & 1 & 0 & 1 & 0 \\
\hline CTA & 1 & 2 & 1 & 2 & 1 & 1 & 2 & & & & & & & \\
\hline SS & $t_{1}$ & $\alpha_{1}$ & $t_{3}$ & $\alpha_{3}$ & $t_{2}$ & $t_{4}$ & $\alpha_{4}$ & $t_{5}$ & $\alpha_{5}$ & $t_{6}$ & $\alpha_{6}$ & $t_{7}$ & $\alpha_{2}$ & $\alpha_{7}$ \\
\hline
\end{tabular}

Figure 3: A sample chromosome.

$S S$ and omit their start times. In this example, (1) only authentication tasks $\alpha_{1}, \alpha_{4}$, and $\alpha_{6}$, are selected to be done by both execution units; (2) computation tasks $t_{2}, t_{4}$, and $t_{7}$ are assigned to execution unit $u_{2}$ while the others are assigned to $u_{1}$; and (3) the assignments for $u_{1}$ and $u_{2}$ are

$$
\begin{aligned}
& \lambda_{1}=\left(t_{1}, s_{t_{1}}\right)\left(\alpha_{1}, s_{\alpha_{1}}\right)\left(t_{3}, s_{t_{3}}\right)\left(\alpha_{4}, s_{\alpha_{4}}\right)\left(t_{5}, s_{t_{5}}\right)\left(t_{6}, s_{t_{6}}\right)\left(\alpha_{6}, s_{\alpha_{6}}\right) \\
& \lambda_{2}=\left(\alpha_{1}, s_{\alpha_{1}}\right)\left(t_{2}, s_{t_{2}}\right)\left(t_{4}, s_{t_{4}}\right)\left(\alpha_{4}, s_{\alpha_{4}}\right)\left(\alpha_{6}, s_{\alpha_{6}}\right)\left(t_{7}, s_{t_{7}}\right)
\end{aligned}
$$

\subsection{Generation of Initial Population}

Each part of a chromosome in the initial population is randomly generated as follows:

- SAT. First, a random binary string of length $|A|$ is generated for each execution unit $u \in \mathbb{U}$. Recall, $S A T_{u}(i)=1$ means that execution unit $u$ partakes in authentication task $\alpha_{i}$. When finishing the generation, only authentication tasks done by 2 or more execution units will be retained. This enforces that $S A T$ satisfies Constraint (10).

- CTA. First, computation tasks are randomly assigned to execution units wrt. their affinity. Next, we check every selected authentication task if one of the execution units partaking in the authentication task performs the respective computation task. If not, we randomly assign the computation task to one of the execution units wrt. their affinity. This enforcement makes the assignment partially satisfy Constraint (11), which additionally requires that an authentication task must be executed right after its respective computation task. Moreover, CTA satisfies Constraints (6) and (7).

- SS. First, we utilize a modification of Kahn's algorithm [6] to topologically sort the authenticatable graph and to enforce that an authentication task must be done right after its corresponding computation task (Constraint (11)). Another challenge in adapting the Kahn's algorithm is to concurrently assign multiple execution units to a single authentication task which may create a deadlock (i.e., one execution unit waits for one other to authenticate computation tasks that they have just concurrently completed). For example, unit $u_{1}$ finished task $t_{1}$ and is waiting for $u_{2}$ to perform authentication task $\alpha_{1}$, while unit $u_{2}$ concurrently finished computation task $t_{2}$ and is also waiting for $u_{1}$ to perform authentication task $\alpha_{2}$. If such situation happens, we will cancel one of the authentication tasks in the cycle and update the SAT. After obtaining the totally ordered sequence, we calculate the start time of each task and form the final SS.

Each newly generated chromosome is checked against those in the population. Two chromosomes are said to be the same if they have the same $S A T, C T A$, and sequence of tasks in SS. If a new chromosome is identical to any in the population, it is discarded, and the generation process is repeated. Otherwise, it will be put into the population. The rationale of obtaining only individual chromosomes is to increase both the diversity and convergence rate.

\subsection{Crossover Operator}

The crossover operator first randomly pick 2 chromosomes from the population and then processes their parts as follows:

- SAT. Recall that an $S A T$ consists of $|\mathbb{U}|$ binary substrings of length $|A|$. The crossover operator randomly generates two cutoff points $c_{s}$ (start point) and $c_{e}$ (end point), such that $c_{s}, c_{e} \in[0,|A|]$ and $c_{s}<c_{e}$. The points divide each substring into three parts, and the middle part of each substring of a $S A T$ will be swapped with the respective middle part of the other SAT. Note, all substrings of a $S A T$ are cut at the same points, so the new $S A T$ s generated by the cross operator still satisfy the Constraint (10).

- CTA. We first apply a similar process as handling SATs with two cutoff points. The swapping can never make the new individuals violate Constraints (6) and (7), but can violate Constraint (11). Therefore, we need to post-process the CTA to ensure that one of execution units partaking in an authentication task does the corresponding computation task.

- SS. We simply invoke our Kahn algorithm, described at the end of Section 6.2, with the new SAT and CTA.

\subsection{Mutate Operator}

The mutate operator first arbitrarily selects a chromosome from the population and then processes its parts as follows:

- SAT. The mutate operator first randomly generates two cutoff points $c_{s}$ and $c_{e}$ as in the crossover operator to divide each substring into three parts. Next, it flips all the bits in the middle part. Finally, it keeps only authentication tasks concurrently assigned to 2 or more execution units.

- CTA. We check every selected authentication task $\alpha$ to enforce that one of the execution units partaking in $\alpha$ performs the respective computation task of $\alpha$ if necessary.

- SS. We simply invoke our Kahn algorithm, described at the end of Section 6.2, with the new SAT and CTA.

\subsection{Selection}

To identify which chromosomes are retained in or removed from the population, each is evaluated by:

- Feasibility, a Boolean value indicating if the chromosome represents a feasible solution or not. An individual is a feasible solution if it satisfies the energy bound.

- CountSAT, an integer whose absolute value is the number of authentication tasks selected. CountSAT is a negative number if the chromosome is an infeasible solution.

- CountPeers, an integer whose absolute value is the total number of peers participating in all selected authentication tasks. CountPeers is a negative number if the chromosome is an infeasible solution.

In order words, (1) each chromosome in the population is associated with a tuple 〈feasibility, CountSAT, CountPeers〉; (2) chromosomes are sorted in descending order wrt. the value of their associated tuples; and (3) only top $P$ individuals will be selected to proceed to the next generation, where $P$ is the size of the population.

\section{ONLINE ALGORITHM}

While the offline algorithms are assumed to be theoretically correct (i.e., execution units should finish their assignments without running out of energy), the physical environment is likely to vary from the expectation set in the energy consumption parameters of tasks 


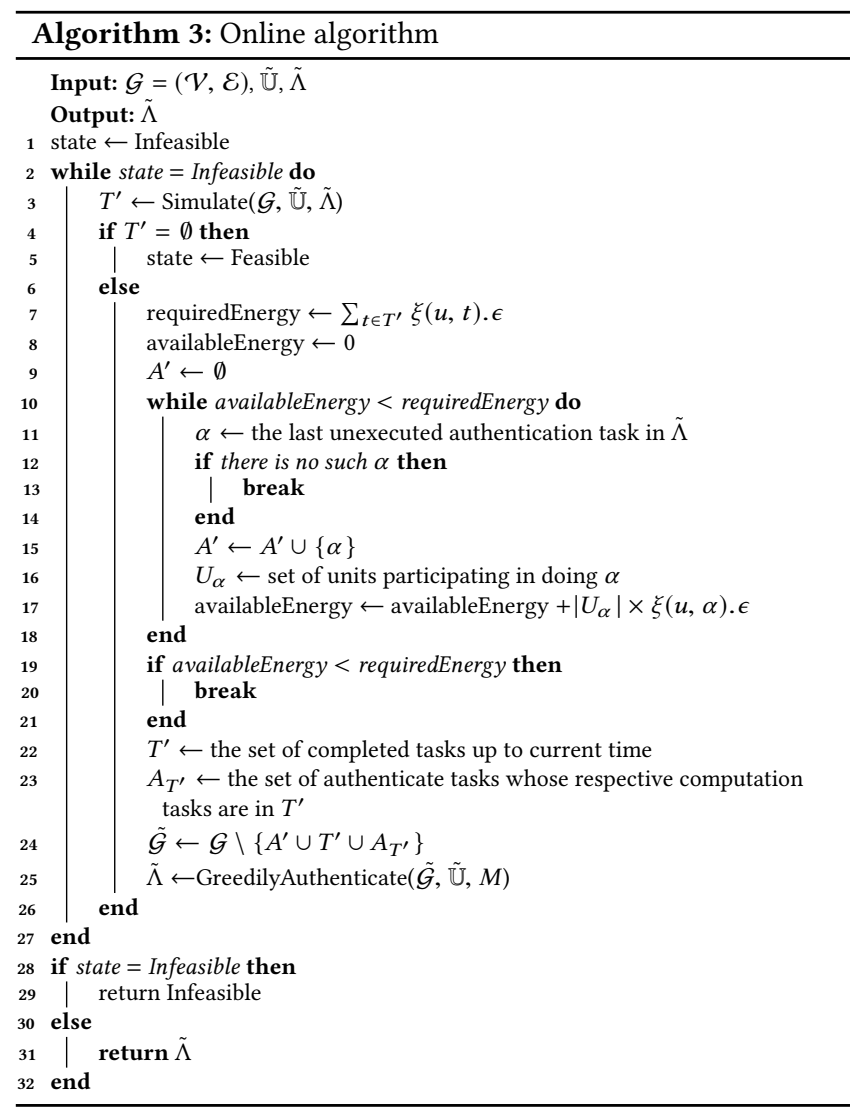

and execution units. For instance, weather conditions can affect the duration and energy consumption of a UAV task, and humidity can affect the battery performance of a wireless sensor. Such changes may result in a reduction of peer-authentication tasks, or more critically, impact the feasibility of the solution (see Constraint 7). We assume that a central entity controls execution units. If an execution unit detects a change in the environment, it informs the central controller which uses our proposed online algorithm to mitigate the change. We propose Algorithm 3 which takes as input an authenticatable task graph, a set of functional execution units, and a set of current assignments to the units. It operates in the following three stages.

\subsection{Simulation}

In the simulation phase (Line 3 of Algorithm 3), the algorithm simulates assignments $\tilde{\Lambda}$. Note that $\tilde{\Lambda}$ does not include assignments for execution units not in $\tilde{U}$. Two scenarios may impact the simulation outcome: (1) a unit may run out of energy before finishing its assignment, and (2) peers participating in authentication may be delayed due to longer than expected computation or transition. Once simulation is complete, we identify the set of computation tasks that were not completed by any execution unit in $\tilde{\mathbb{U}}$. If this is an empty set, our algorithm returns success indicating that the current set of assignments $\tilde{\Lambda}$ will result in a feasible solution. Otherwise, we denote the set of incomplete computation tasks as $T^{\prime}$ and proceed to the next step.

\subsection{Backtracking}

In this step, our objective is to identify authentication tasks to eliminate from assignments $\tilde{\Lambda}$ to free up more energy for executing the remaining computation tasks in $T^{\prime}$. A simple backtracking heuristic first identifies the minimum amount of energy required to execute the remaining tasks (Line 7). This is simply the sum of energy consumption of all tasks in $T^{\prime}$. This is a lower bound on the actual energy required to execute the remaining tasks which could be higher due to transition costs. At this moment we do not know which transitions will be taken and so we assume all transitions cost zero energy. Subsequently, the heuristic backtracks from the last authentication task in $\tilde{\Lambda}$, adding up freed up energy until it is greater than or equal to the minimum amount of energy required to execute the remaining tasks (Line 17).

The output of this step is a set of authentication tasks $A^{\prime}$ which will be removed from $\tilde{\Lambda}$. The current time refers to the time the central entity has received a notification of a change in the environment and invoked the online algorithm to reevaluate the current plan. If we cannot get enough freed up energy, this implies that there are no more authentication tasks to remove to improve coverage of computation tasks, possibly because all other authentication tasks have been executed already. In this case, we terminate the online algorithm with a failure due to infeasibility.

\subsection{Calibration}

In this step, we modify assignments $\tilde{\Lambda}$ to optimize for coverage of computation tasks by simply calling the greedy authentication algorithm. In order to accelerate the algorithm, we invoke the algorithm on the modified graph which contains only unexecuted computation tasks and possibly executable authentication tasks (Line 25).

\section{EVALUATION}

To rigorously analyze the techniques presented in the previous section, we target the multi-UAV example discussed in the previous sections. Figure 4 illustrates a sample authenticatable task graph of a $2 \times 3$ grid. Each grid cell contains (1) a computation task (e.g., for scanning or detecting a certain type of object or anomaly), and (2) an authentication task performed by two or more UAVs (Formula (10)), which verifies the output of the computation task either by corroborating the result or authenticating peers. The gray circle denotes the depot from which all UAVs must take off and does not require a corresponding authentication task (i.e., the depot is the dummy start task $d_{s}$ introduced in Section 4). The solid arrows represent transitions based on task dependencies. That is, all UAVs must take off from the depot before flying to any cell, and the cells can be scanned in any order. The dashed lines represent the transitions from computation tasks to the corresponding authentication task (Equation (2)). The amounts of time and energy required by a UAV to do a computation task are determined by execution cost function $\xi$, while those for flying from one cell to another one are determined by transition function $\mathcal{T}$.

A UAV is an execution unit $u$ that accomplished a sequence of computation as well as authentication tasks. Moreover, the assignment in $\lambda_{u}^{S}$ is identified such that the problem constraints identified in Section 3.1 are satisfied and the optimization objectives in Section 3.2 are maximized. This, in turn, means that by solving our optimization problem, we will obtain a flight path for each UAV that maximizes the reliability of the output while staying within the 


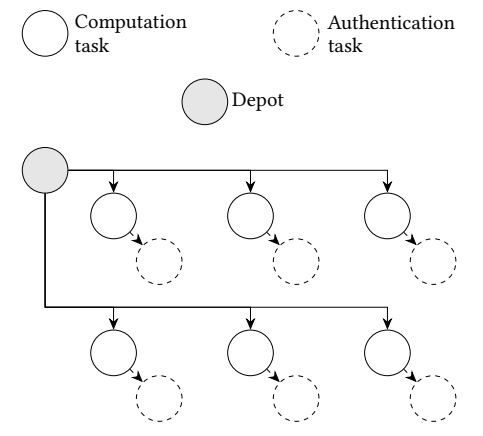

Figure 4: Authenticatable task graph of UAVs exploring a $2 \times 3$ grid map.

energy bounds of the UAV's batteries. Observe that our proposed model also allows encoding variability in the map as well as the capability of UAVs.

\subsection{Simulations}

8.1.1 Analysis of Offline Algorithms. Figure 5 shows the results of our simulations of the UAV system. We ran the MILP-based algorithm for the $3 \times 3$ grid to serve as a reference for what an optimal set of flight paths can achieve. For larger grids, MILP does not scale well. As expected, the MILP outperforms the genetic algorithm (GA) and the greedy algorithm in terms of number of authentication tasks (see Fig. 5a). The MILP also consumes more energy, utilizing the battery almost fully to maximize authentications.

Our simulations show that the GA consistently outperforms the greedy algorithm. The greedy algorithm always makes the cheapest choice which may not be beneficial in the long run. This is the heart of the problem that our work tackles: how security decisions can non-immediately impact resource consumption and system objectives. Figure $5 \mathrm{~b}$ shows that the GA utilizes the battery more efficiently. Note that NA refers to an algorithm that does not authenticate tasks at all. Figure $5 \mathrm{~d}$ shows that there is a case where the greedy algorithm uses more energy than the genetic algorithm. This does not translate into more authentications (Figure 5c) since the greedy algorithm is making choices that incur more travel time costs in the future.

Moreover, the genetic algorithm is more inclined to schedule authentications at random points in time, further obscuring the authentication process from an adversary. In the next section, we discuss simulation details of the online algorithm which is based on the genetic algorithm. Figure 6 demonstrates how authentication tasks are randomly placed. This is opposed to the greedy algorithm that performs all authentications at the beginning.

8.1.2 Analysis of the Online Algorithm. In order to analyze our online algorithm, we design the following scenarios. In the first scenario, four UAVs explore a $5 \times 5$ grid. If the conditions during the entire operation do not change, then the UAVs complete their mission in $381 \mathrm{~s}$ and accomplish 9 peer-authentications (see Fig.6). This solution is obtained by the offline genetic algorithm. Now, we take the same flight paths and inject a UAV crash at time 170s. The online algorithm realizes that the current flight paths are not feasible (due to reduction in the current energy bound) and computes a new schedule, which results in new flight paths and authentication tasks for the remaining three UAVs. The new flight paths results in a total of 5 peer-authentications. In the third scenario, we inject an additional UAV crash at time 185s. The drop in the total energy bound results in a situation where no further peer-authentication can be achieved by the remaining to UAVs after the second crash.

\subsection{Experiments with Real Multi-UAV Network}

We now report the results of implementation and deployment of our algorithms in a real network of UAVs. The experimental platform consists of a motion capture system (12 OptiTrack infrared cameras) in 480 square feet lab space (see Fig. 7). We employed Intel Aero drones and programmed the PX4 flight controller. The UAVs communicate with the base station over a dedicated WiFi TCP/IP possibly network. Our goal is to demonstrate that our online algorithm can react effectively to uncertainties caused by the physical environment.

Our experiment is on a $4 \times 4$ grid with two UAVs that are supposed to cover all 16 cells of the grid, where one UAV flies in a higher altitude. The genetic algorithm identifies flight paths with 16 computation tasks and 16 peer-authentications within the $16 \mathrm{~J}$ battery energy bound of the UAVs. When the UAVs follow the flight paths prescribed by the genetic algorithm, they end up completing only 11 computation and 11 authentication tasks due to reaching their energy limit too quickly, resulting in a pre-mature completion of the mission. The reason for this is that the top UAV creates spiral air flow, causing the lower UAV to spend about $12 \%$ more energy than predicted by the genetic algorithm.

Next, we ran our online algorithm initialized by the same flight paths as the genetic algorithm. The online algorithm effectively conducted appropriate re-planning and executed 16 computation tasks (i.e., covering the entire grid) and 9 authentication tasks. That is, the online algorithm successfully managed the security-energy tradeoff within the energy limits of the UAVs ${ }^{1}$.

\section{RELATED WORK}

Security of CPS has been a topic of increasing importance in the last several years. The work in $[1,10]$ gives insight into the type of attacks that CPS will be specifically subject to. The work in [5] builds a taxonomy of cyber-physical security research, showing a classification of attacks and controls for different types of CPS.

Most of the controls discussed in the literature focus on adapting state-of-the-art security techniques to fit within CPS constraints. The literature tackles several aspects of cyber security. The work in [8] covers different time critical intrusion detection mechanisms usable in a CPS setting. Other work focused on proposing security aware alternatives to traditional CPS insecure communication [4]. Out of band authentication was introduced in the scope of medical devices [9]. The work in [3,11] introduces hardware-based cryptography solutions specifically designed for smart cars.

One approach relative to the work in this paper is scheduling security tasks in CPS. The work in [7] targets the problem of determining when to inject cryptographic checks without interfering with control tasks. The paper proposes an approach that tries to maximize security checks while maintaining a predefined level of control quality. Similarly, the work in [12] proposes a feedback scheduling technique for maintaining network QoS in wireless sensor networks. Both fall under soft real-time constraints, where essentially security is traded off with deadline adherence.

\footnotetext{
${ }^{1}$ The video clip of this experiment can be found at http://www.cs.iastate.edu/ borzoo/ iccps19.
} 


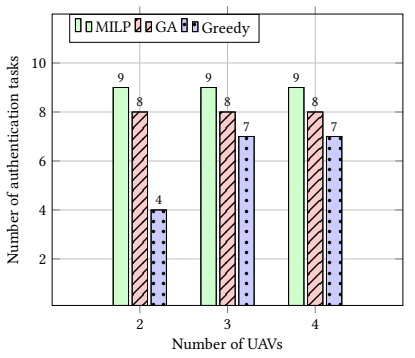

(a) $3 \times 3$ Authentication

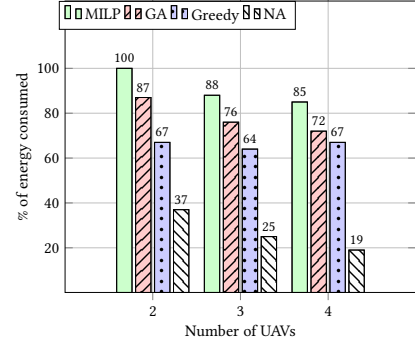

(b) $3 \times 3$ Energy Consumption

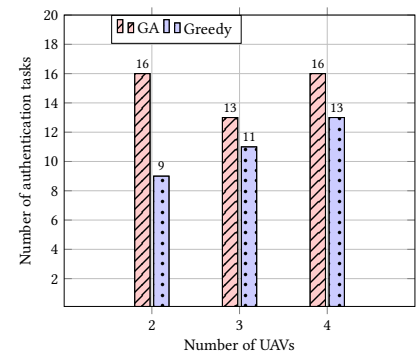

(c) $4 \times 4$ Authentication

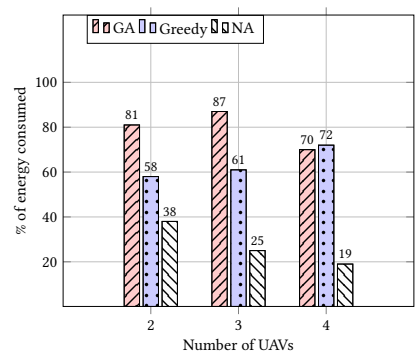

(d) $4 \times 4$ Energy Consumption

Figure 5: Simulation results for $3 \times 3$ and $4 \times 4$ grids.

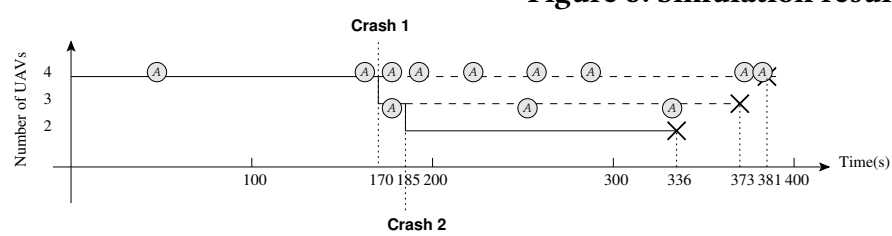

Figure 6: Simulation scenarios for the online algorithm.

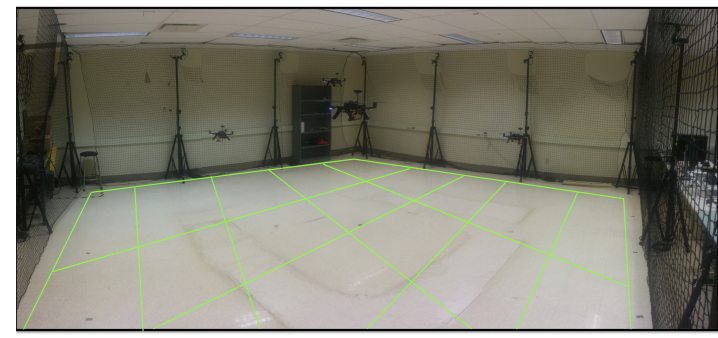

Figure 7: Experimental platform.

In our work, we tackle the tradeoffs between security and energy consumption. We propose a model that accounts for how security tasks can potentially change the control flow of a distributed system. Our proposed algorithms provide a mechanism of controlling the frequency of authentication in a dynamic environment while maintaining energy bounds.

\section{CONCLUSION AND FUTURE WORK}

In this paper, we investigated the security-energy tradeoff issues in distributed CPS. We proposed a general-purpose model called authenticatable task graphs that capture the interactions of the security vs. energy and allow system designers to parameterize the tradeoffs in their system. That is, authentication tasks can be intermittently and arbitrarily inserted into the computation task graph. We also introduced three different offline techniques that identify optimal and near-optimal schedules for execution units to compute functional tasks as well as peer-authentication tasks, where units verify the sanity of the output of each other to provide resilience against cyberattacks. We also introduced an online algorithm that reacts to situations, where the physical environment invalidates some of the design, implementation, or optimization assumptions. We reported results of simulations of all of our algorithms as well as a proof of concept using a real network of UAVs that carry our a joint search mission.
Although our proposed online algorithm can dynamically adjust the assignments in responding to changes of the physical environment, it is a bottleneck, since it has to deal with all execution units. Thus, an interesting and practical problem is to design an online decentralized algorithm, where execution units make their scheduling decisions through communicating with each other. A more complicated scenario to investigate is where the authenticatable task graph is dynamic, i.e., tasks may join and leave during the execution. Another important line of research is where execution units may become Byzantine, i.e., they non-intentionally or maliciously misrepresent their observation or peer-authentication results.

\section{ACKNOWLEDGEMENT}

This work is partially sponsored by the NSF SaTC-1813388 and a grant from Iowa State University.

\section{REFERENCES}

[1] Alvaro Cardenas, Saurabh Amin, Bruno Sinopoli, Annarita Giani, Adrian Perrig, Shankar Sastry, et al. 2009. Challenges for securing cyber physical systems. In Workshop on future directions in cyber-physical systems security, Vol. 5.

[2] Thomas H Cormen, Charles E Leiserson, Ronald L Rivest, and Clifford Stein. 2009. Introduction to algorithms. MIT press.

[3] R Escherich, I Ledendecker, C Schmal, B Kuhls, C Grothe, and F Scharberth. 2009. SHE: Secure Hardware Extension-Functional Specification, Version 1.1. Hersteller-Initiative Software (HIS) AK Security (2009).

[4] Igor Nai Fovino, Andrea Carcano, Marcelo Masera, and Alberto Trombetta. 2009. Design and implementation of a secure modbus protocol. In International conference on critical infrastructure protection. Springer, 83-96.

[5] Abdulmalik Humayed, Jingqiang Lin, Fengjun Li, and Bo Luo. 2017. Cyberphysical systems security - A survey. IEEE Internet of Things fournal 4, 6 (2017), 1802-1831.

[6] Arthur B Kahn. 1962. Topological sorting of large networks. Commun. ACM 5, 11 (1962), 558-562.

[7] Vuk Lesi, Ilija Jovanov, and Miroslav Pajic. 2017. Security-Aware Scheduling of Embedded Control Tasks. ACM Trans. Embed. Comput. Syst. 16, 5s, Article 188 (Sept. 2017), 21 pages. https://doi.org/10.1145/3126518

[8] Robert Mitchell and Ing-Ray Chen. 2014. A survey of intrusion detection techniques for cyber-physical systems. ACM Computing Surveys (CSUR) 46, 4 (2014), 55.

[9] Michael Rushanan, Aviel D Rubin, Denis Foo Kune, and Colleen M Swanson. 2014. Sok: Security and privacy in implantable medical devices and body area networks. In 2014 IEEE Symposium on Security and Privacy (SP). IEEE, 524-539.

[10] Lui Sha, Sathish Gopalakrishnan, Xue Liu, and Qixin Wang. 2008. Cyber-physical systems: A new frontier. In Sensor Networks, Ubiquitous and Trustworthy Computing, 2008. SUTC'08. IEEE International Conference on. IEEE, 1-9.

[11] Marko Wolf and Timo Gendrullis. 2011. Design, implementation, and evaluation of a vehicular hardware security module. In International Conference on Information Security and Cryptology. Springer, 302-318.

[12] Feng Xia, Longhua Ma, Jinxiang Dong, and Youxian Sun. 2008. Network QoS management in cyber-physical systems. In Embedded Software and Systems Symposia, 2008. ICESS Symposia'08. International Conference on. IEEE, 302-307. 